\title{
Comparative Effectiveness and Safety of 32 Pharmacological Interventions Recommended by Guidelines for COVID-19: A Systematic Review and Network Meta-Analysis Combining 66 Trials
}

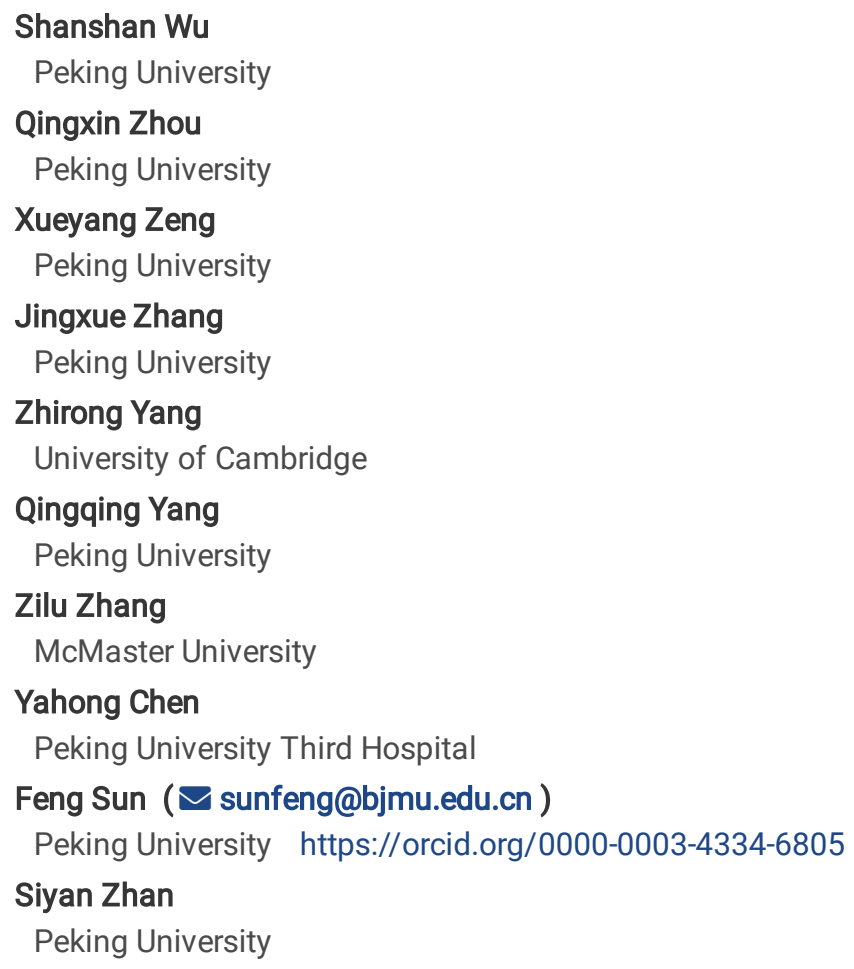




\section{Abstract}

Background: To evaluate comparative efficacy and safety of pharmacological interventions in patients with coronavirus disease 2019.

Methods: Medline, Embase, the Cochrane Library and clinicaltrials.gov were searched for randomized controlled trials (RCTs) in patients infected with SARS-COV-2/SARS-COV. Random-effects network meta-analysis within Bayesian framework was performed, followed by Grading of Recommendations Assessment, Development and Evaluation (GRADE) system assessing quality of evidence. The primary outcome of interest includes mortality, cure, viral negative conversion (VNC) and overall adverse events (OAE). Odds ratio (OR) with 95\% confidence interval $(\mathrm{Cl})$ was calculated as the measure of effect size.

Results: 66 RCTs with 19,095 patients were included, involving standard care (SOC), 8 different antiviral agents, 6 different antibiotics, high and low dose chloroquine (CQ_HD, CQ_LD), traditional Chinese medicine (TCM), corticosteroids and other treatments. Compared with SOC, significant reduction of mortality was observed for TCM ( $O R=0.34,95 \% \mathrm{Cl}: 0.20-0.56$, moderate quality) and corticosteroids ( $O R=0.84,0.75$ 0.96 , low quality) with improved cure rate $(\mathrm{OR}=2.16,1.60-2.91$, low quality for $\mathrm{TCM} ; \mathrm{OR}=1.17,1.05-1.30$, low quality for corticosteroids). However, increased risk of mortality was found for CQ_HD versus SOC (OR=3.20, 1.18-8.73, low quality). TCM was associated with decreased risk of OAE (OR=0.52, 0.38-0.70, very low quality) but CQ_HD (OR=2.51, 1.20-5.24) and IFN $(\mathrm{OR}=2.69,1.02-7.08)$ versus SOC with very low quality) were associated with an increased risk.

Conclusions: Corticosteroids and TCM may reduce mortality and increase cure rate with no increased risk of OAEs compared with standard care. CQ_HD might increase the risk of mortality. CQ, IFN and other antiviral agents could increase the risk of OAEs. The current evidence is generally uncertain with low quality and further high-quality trials are needed.

\section{Introduction}

As of 3 September 2020, more than 25.8 million people have been infected with severe acute respiratory syndrome coronavirus 2 (SARSCOV-2), which has similar genetics to SARS-COV ${ }^{1}$. This global pandemic coronavirus disease 2019 (COVID-19) has caused 859,130 deaths in 216 countries, which has become a major public health problem and presents unprecedented challenge ${ }^{1}$.

So far, many kinds of drugs in addition to standard of care (SOC) are recommended by different clinical guidelines ${ }^{2-5}$, including antiviral agents, immune-based therapies [such as corticosteroids (COR), convalescent plasma (CON_PLA) and interferons (IFN)], hydroxychloroquine or chloroquine (CQ), traditional Chinese medicine (TCM) and other adjunctive therapies. However, no specific drugs were currently proven and nearly all drugs are off-label prescribed ${ }^{6}$. Despite numerous on-going or finished clinical trials, substantial uncertainty about effectiveness and safety still exists in these therapies owing to limited sample size and large variability with insufficient power.

Although several meta-analyses ${ }^{7-13}$ have been available, most are pairwise comparisons between two kinds of drugs with few studies included or only narrative synthesis. Moreover, methodological limitations exist in most meta-analyses, such as combining observational studies with RCTs, mistaking cohort studies as RCTs, mixing controlled groups and omitting searching Chinese databases ${ }^{14-17}$. Additionally, these pairwise meta-analyses are not able to provide evidence on the comparative effectiveness and safety for all available treatments. While several network meta-analyses (NMA) are ongoing ${ }^{18-20}$, no results for the comprehensive assessment have been reported, and some treatments such as TCM and blood products are not included in these NMA. Furthermore, their network is sparse without the inclusion of similar genetic SARS-COV studies. Besides, placebo and SOC are considered as a single treatment in these NMA which may omit the potential placebo effect ${ }^{21}$.

Therefore, we aimed to collect all RCTs comparing any kinds of pharmacological interventions with placebo or SOC among SARS-COV-2 and SARS-COV patients and conduct an NMA to assess comparative efficacy and safety for these treatments.

\section{Methods}

This study was registered on International Prospective Register of Systematic Review PROSPERO, number CRD42020168178. The study was conducted according to PRISMA-NMA checklist.

\section{Data sources and Searches}

PubMed, Embase, Web of Science, Cochrane Central Register of Controlled Trials and three Chinese databases including SinoMed, China National Knowledge Infrastructure (CNKI) and WanFang Database were searched from inception to July 20 th, 2020 (Appendix 1 for full 
details about search strategy). In addition, we also checked reference list of all relevant articles to identify additional studies.

\section{Study selection}

Only RCTs written in English or Chinese with available outcome data in which different pharmacological interventions compared in patients with suspected or confirmed SARS-COV-2 or SARS-COV were included. Pharmacological interventions were defined according to recommended guidelines ${ }^{[2-5]}$, including antiviral agents [Ribavirin (RIB), lopinavir/ritonavir (LPV_RIT), Remdesivir (REM), Baloxavir marboxil (BAL), Favipiravir (FAV), Umifenovir (UMI), Azvudine (AZV) and darunavir/cobicistat (DRV_c)], antibiotics [Azithromycin (AZI), Lincocin (LIN), Fluoroquinolone (FLU), Cefperazone-Sulbacta (CEFs), Levofloxacin (LEV) and Quinolone (QUI)], COR, CQ, TCM, CON_PLA, a-Lipoic acid (ALA), Ruxolitinib (RUX), Novaferon (NOV), colchicine (COL), IFN, Octagam (IVIG), SOC and placebo (PLA). The primary outcomes of interest included mortality, cure rate, viral negative conversion and overall adverse events rate (OAE). Secondary outcomes included diarrhea (DIA), acute kidney injury (AKI), transaminase elevation (TE), secondary infection (SEI), heart failure (HF), acute respiratory dyspnea syndrome (ARDS), serious $A E$ (SAE), hospitalization duration (HD) and time to fever resolution (TFR). The eligibility of studies for inclusion criteria was assessed independently by six reviewers (XMC, XYT, SC, XYZ, JXZ and QXZ) in duplicate. Any discrepancies were resolved by consensus between other independent reviewers (FS, JXZ and QXZ).

\section{Data extraction and quality assessment}

Data were extracted with respect to trial information (author, publication year, country, virus type, preprint or not, guideline or not, sample size, trial duration, types of intervention and control), population characteristics [mean age \pm SD (standard deviation), proportion of female, disease severity], reported outcomes (number of events for dichotomous outcomes and mean with SD for continuous outcomes) and information on methodology. Four investigators (FS, XYZ, JXZ and QXZ) extracted data independently in duplicate. Risk of bias was assessed according to Cochrane risk of bias tool (ROB tool) ${ }^{22}$. Additionally, GRADE (The Grading of Recommendations Assessment, Development, and Evaluation) framework was used to assess quality of evidence contributing to each network estimation, which characterizes the quality of a body of evidence on the basis of study limitations, imprecision, inconsistency, indirectness and publication bias $^{23}$

\section{Data Synthesis and Analysis}

\section{Methods for direct treatment comparisons}

Pairwise meta-analysis was performed using DerSimonian-Laird random-effects model. Odds ratio (OR) and weighted mean difference (WMD) with 95\% confidence interval (Cl) were calculated as effect measures for dichotomous and continuous outcomes respectively. The $R^{2}$-statistic was calculated as a measure of proportion of overall variation that is attributable to between-study heterogeneity. For studies with zero-event in both arms, a continuity correction of 0.5 was used ${ }^{24}$. Besides, subgroup pairwise meta-analysis was conducted according to different virus type (SARS-COV-2 and SARS-COV).

\section{Methods for indirect and mixed comparisons}

A random-effects NMA within Bayesian framework ${ }^{25}$ was then performed by 100,000 iterations with 20,000 adaptations and thinner equals to 10 . OR and WMD with $95 \% \mathrm{Cl}$ were summarized for dichotomous and continuous outcomes respectively. We estimated ranking probabilities for all treatments of being at each possible rank for each intervention. The treatment hierarchy was summarized and reported as surface under the cumulative ranking curve (SUCRA), mean ranks and rank heat plot ${ }^{26}$.

\section{Examination of assumptions in NMA (consistency, transitivity and heterogeneity)}

To check the assumption of consistency in the entire analytical network, a design-by-treatment approach was used ${ }^{27}$. The node splitting method were used to assess inconsistency of model with separating evidence on a particular comparison into direct and indirect evidence. Heterogeneity was assessed with common tau ${ }^{2}$ statistic and predictive interval ${ }^{28}$. The transitivity assumption was evaluated by comparing distribution of clinical variables which could act as effect modifiers across treatment comparisons. Contribution table was used to assess the contribution of each direct comparison to the estimation of each network meta-analytic summary effect ${ }^{28}$. Additionally, comparisonadjusted funnel plot was used to detect potential publication bias for outcomes with at least 10 trials.

All analyses were conducted using R 4.0.2 (gemtc package for NMA and node-split analysis; ggplot2 package for network evidence plot, forest plots and cumulative rank probability graphs; netmeta package for funnel plot; fields package, RColorBrewer package and circlize 
package for rank heat plot), STATA 13.0 (pairwise meta-analysis, estimation of local heterogeneity and contribution plot) and CINEMA website (https://cinema.ispm.unibe.ch/ for GRADE results).

\section{Patient and public involvement}

Patients and the public were not involved in this review.

\section{Results \\ Study characteristics}

Of 45778 studies retrieved from the searchers, 66 trials with 32 treatments (19, 095 patients) met inclusion criteria (Fig. 1), including 8 different antiviral agents, 6 antibiotics, high and low dose CQ (CQ_HD, CQ_LD), 5 add-on treatments (LPV_RIT_IFN, LPV_RIT_RIB_IFN, RIB_IFN, NOV_LPV_RIT, CQ_LD_AZI), COR, TCM, CON_PLA, ALA, RUX, NOV, COL, IFN, IVIG, SOC and PLA. Among these treatments, 6 antibiotics were separately compared in 1 trial for SARS-COV ${ }^{[29]}$ and 1 trial for SARS-COV-2 ${ }^{[30]}$, which could not be connected with other treatments in the trial network. Excluding the six antibiotics, 26 treatments within 64 trials were finally analyzed in the network.

90.6\% (58/64) of trials were two-arm studies and only 6 were multiple-arm studies (Appendix 2). Overall, 18,881 patients were involved in the meta-analysis, of whom, 17,416,14,708, 1,197 and 11,698 patients contributed to four outcomes of mortality, cure rate, VNC and OAE, respectively (Fig. 2). Appendix 2 summarized the characteristics of included trials. Publication year were focused from 2003 to 2005 for SARS-COV (19 trials) and 2020 for SARS-COV-2 (45 trials). Trial duration ranged from 5 to 75 days with a median duration of 15 days [interquartile range (IQR): 13-28 days]. The mean age of included patients was 48.9 years [standard deviation (SD) 9.8] and mean proportion of female was $45.7 \%$ (IQR:40\%-53.5\%). Disease severity was mild/moderate in 23 trials and moderate/severe in the others. SOC, TCM, antiviral agents and CQ were the most common studied drugs, within51, 31, 20 and 14 trials, respectively, followed by PLA (6 trials) and $\operatorname{COR}$ (4 trials).

\section{Methodological quality and risk of bias results}

Among the 66 included trials, allocation concealment and blinding of participants and personnel were not clearly reported in $84.8 \%$ and $48.5 \%$ of the cases, respectively. By contrast, methods for randomization and incomplete outcome data were appropriately described in large majority of studies ( $56.1 \%$ and $86.4 \%$, respectively). $31.8 \%$ of trials were open label and $72.7 \%$ did not have selective reporting (the remaining $19.7 \%$ was unclear due to no related protocol). Additionally, $12.1 \%$ of trials were funded by company and $43.9 \%$ did not report funding sources (Appendix 3). Overall, risk of bias across evidence network was relatively low.

\section{Results of pairwise meta-analysis}

The effects of different drugs on mortality, cure rate, VNC and OAE from pairwise meta-analyses are shown in Appendix 4. TCM and COR were associated with a significant reduction in mortality $(\mathrm{OR}=0.33,95 \% \mathrm{Cl}: 0.20-0.55$ and $\mathrm{OR}=0.85,0.75-0.96$ respectively) and a significant increase in cure rate $(\mathrm{OR}=2.17,1.61-2.92$ and $\mathrm{OR}=1.17,1.05-1.30$ respectively) compared with SOC. Compared with CQ_LD, CQ_HD showed an evident increase in mortality $(O R=3.33,1.19-9.35)$ ). Compared with SOC, TCM showed a significant decrease in the risk of $\mathrm{OAE}(\mathrm{OR}=0.52,0.38-0.71))$, whereas CQ_HD, IFN, COL and CQ_LD_AZI were all associated with increased risk, with the ORs ranging from 1.65(1.11-2.47,CQ_LD_AZI) to 3.83 (1.68-8.70,CQ_HD). CQ_LD was significantly associated with increased risk of OAE versus placebo $(\mathrm{OR}=2.75,1.80-4.20)$.

\section{Results of NMA}

Results of NMA are reported in Fig. 3-4. Significant decrease in mortality was observed for TCM $(O R=0.34,0.20-0.56)$ and COR (OR = $0.84,0.75-0.96)$ versus SOC, while CQ_HD could significantly increase the risk $(O R=3.20,1.18-8.73)$. Besides, a significant reduction in mortality was detected for TCM versus COR, CQ_HD and CQ_LD with the ORs.

Regarding cure rate, the pooled results favor TCM $(O R=2.16,1.60-2.91)$ and COR $(O R=1.17,1.05-1.30)$ in comparison with SOC. When compared with COR and CQ_LD, TCM could significantly improve cure rate with an OR of 1.85(1.35-2.56) and 2.38(1.72-3.33), respectively. No significant results were observed on VNC for any comparison.

In terms of OAE, both TCM $(\mathrm{OR}=0.52,0.38-0.70)$ and REM_10 $(\mathrm{OR}=0.31,0.19-0.52)$ were associated with decreased risk, whereas COL, CQ_HD and IFN were associated with increased risk versus SOC, with the ORs ranging from 2.51 (1.20-5.24, CQ_HD) to 3.81 (1.55-9.29, COL). In comparison with COL, COR, CQ_HD, CQ_LD, IFN and LPV_RIT, a significant reduction of OAE was found for TCM). 
Results of NMA for secondary outcomes are listed in Appendix 5. Among separate AEs, a significant increased risk of diarrhea was detected for COL, CQ_HD, CQ_LD, LPV_RIT and LPV_RIT_RIB_IFN versus SOC (OR ranging from 3.80(1.55-9.26, COL) to 9.62(1.70-56.11, LPV_RIT)) and TCM (OR ranging from 8.77(2.99-25.74, COL) to 22.27(3.53-142.16, LPV_RIT)). Meanwhile, TCM showed significantly decrease in the risk of diarrhea $(O R=0.43,0.24-0.79)$ and $S E I(O R=0.33,0.17-0.64)$ when compared with SOC. Additionally, an evident reduction of ARDS was found for $L P V \_R I T$ versus SOC $(O R=0.37,0.18-0.80)$. No other significant results were detected for other separate AEs (AKI, SAE, TE and HF).

TCM, RIB_IFN, CON_PLA, LPV_RIT_RIB_IFN and LPV_RIT were all associated with shorter hospitalization length ranging from - 17.80 $(-29.92,-5.74$, RIB_IFN) to $-2.53(-3.43,-1.63$, TCM $)$ days when compared with SOC. Furthermore, TCM could reduce hospitalization length for 2.63(CQ_LD) to 5.09(IFN) days versus CQ_LD, CQ_LD_AZI and IFN. Besides, a significant reduction on TFR was found for TCM versus SOC (WMD $=-1.03$ days, 95\%Cl: $-1.09,-0.97)$.

According to contribution tables of the network (Appendix 6), comparison of SOC versus antiviral agents, COR and CQ had the largest contribution in all 4 entire networks for primary outcomes with $51.8 \%, 56.5 \%, 56.9 \%$ and $50.0 \%$ for mortality, cure rate, VNC and OAE, respectively.

\section{Transitivity, inconsistency and heterogeneity}

Assessment of transitivity by box plots indicated mean age and proportion of female across treatment comparisons were relatively similar (Appendix 7). The test for global inconsistency did not detect any significant difference between consistency and inconsistency models for all the 4 primary outcomes $(p=0.994$ for mortality, $p=0.763$ for cure, $p=0.952$ for VNC and $p=0.773$ for OAE, respectively) and 3 secondary outcomes $(p=0.984$ for DIA, $p=0.845$ for SAE and $p=0.614$ for TE, respectively), except for other 6 secondary outcomes(AKI, ARDS, HD, HF, SEl and TFR) could not conduct consistency test due to no loop in the whole network. The test for inconsistency from nodesplitting model showed no significant difference in all comparisons across all outcomes (Appendix 8). Most comparisons in all 13 outcomes were with low heterogeneity as indicated in the predictive interval. At visual inspection, funnel plots for all 7 outcomes with number of studies greater than 10 (Appendix 9) were symmetric and did not suggest any significant risk of publication bias.

\section{Rank heat plot and SUCRA of all treatments}

Figure 5 and Appendix 10 showed the mean values of SUCRA for providing the hierarchy ranking of different treatments on all 13 outcomes. Due to sparse network data and non-definitive results in most comparisons, the ranking might be biased and interpretation should be made with caution.

\section{GRADE evaluation on quality of evidence}

According to GRADE, the quality of evidence ranged between very low and moderate (Appendix 11). In terms of TCM versus SOC, the quality was moderate for mortality, low for cure rate, and very low for VNC and OAE. As for COR versus SOC, the quality was low for mortality, cure rate and OAE while very low for VNC. Regarding to CQ_HD versus SOC, the quality was low for mortality and very low for OAE.

\section{Subgroup analyses}

In addition, subgroup pairwise meta-analysis by virus type confirmed the beneficial effect on mortality and cure rate of TCM and COR versus SOC, reduction effect on OAE of TCM versus SOC, increased risk of mortality for CQ_HD versus CQ_LD, and increased risk of OAE for CQ_HD, IFN and CQ_LD_AZI versus SOC in SARS-COV-2, which were in agreement with those previous produced (Appendix 12).

\section{Discussion}

Considering the global pandemic of COVID-19, increasing attention is being paid to the effectiveness and safety of pharmacological treatments. Our NMA with 66 trials and 19,095 patients suggested that corticosteroids and TCM could probably reduce mortality and increase cure rate with no increased risk of OAEs compared with SOC. However, CQ_HD might increase the risk of mortality. CQ, IFN and other antiviral agents could increase the incidence of OAEs.

In line with other studies 7,9,18,31-35, we did not find any potential effect on reducing mortality or increasing cure/viral clearance rate for IFN and any antiviral agents, but rather we found CQ_HD was associated with increased mortality. It should be recognized that several sideeffects may be caused according to some observational studies and trials, such as QT prolongation by CQ and FAV, gastrointestinal complications by LPV_RIT and UMI ${ }^{18,31-35}$. In our study, an increased risk of OAE was detected for CQ and IFN, and diarrhea for LPV_RIT and LPV_RIT_RIB_IFN, although no other significant risk of OAE was detected for antiviral agents. Given the potential harms with lack of effectiveness, they are not recommended by several guidelines as treatment for COVID-19, particularly for mild to moderate patients ${ }^{2-5}$. For 
severe patients who need supplemental oxygen or intensive care, REM is weakly recommended to shorten the time to clinical improvement

5 . However, the evidence was low- or very low-quality with no observed effect on hospitalization duration and mortality.

According to the $\mathrm{WHO}$ and other guidelines, routine use of systematic corticosteroids was not recommended for treatment of viral pneumonia, except for patients who require supplemental oxygen and mechanical ventilation ${ }^{2-4}$. A systemic inflammatory response may develop in patients with severe COVID-19, which could result in lung injury and multisystem organ dysfunction. Since corticosteroids could decrease inflammatory response, it might lead to fewer intensive care unit transfers, thereby lowering mortality rate. Several studies, including RECOVERY trial ${ }^{36}$ and meta-analyses ${ }^{13,18}$, show similar findings. However, in mild or moderate patients, this benefit may be outweighed by adverse effects such as delayed viral clearance and increased risk of secondary infection. Results from different studies are not consistent. A meta-analysis ${ }^{37}$ of 6458 patients with influenza pneumonia indicated $75 \%$ and $98 \%$ increase in mortality and secondary infection risk respectively, while a retrospective study ${ }^{38}$ of 201 patients with COVID-19 found $62 \%$ decreased risk of mortality for methylprednisolone. Our NMA found improved effects of corticosteroids on mortality and cure rate and no effects on VNC and SEI versus SOC, perhaps due to severe pneumonia of included patients with older age (mean age $=66.2$ ). Further subgroup and meta-regression NMA according to disease severity are essential upon the completion of many other on-going trials, since the inclusion of mild, moderate and severe patients may dilute the effect of corticosteroids.

Our study found TCM as adjuvant therapy achieved significant lower mortality and OAE with higher cure rate, which is consistent with previous meta-analysis ${ }^{14-16}$. While use of traditional herbs remain controversial in clinical practice, the beneficial effect is biologically reasonable ${ }^{39-43}$. The most commonly used herbs were Radix Glycyrrhizae (Gancao), Astragali Radix (Huangqi), Rhizoma Pinelliae Tematae (Banxia) and Forsythiae Fructus (Lianqiao), which could clear away heat and toxic material, eliminate phlegm-dampness and replenish qi according to TCM theory ${ }^{39}$. Moreover, it has also been confirmed that these herbs have a wide of pharmacological effects including anti-inflammatory, antiviral, antipyretic, antioxidative and immunoregulatory effects ${ }^{40-43}$. Thus, it could maintain the homeostasis of immune system, inhibit a variety of viruses and thereby effectively block the ranging from mild to critical. However, the dosage, composition, treatment duration and disease severity of COVID-19 cases should be taken into account when considering TCM, since these factors are closely related to safety issues. Despite lower AE rate in TCM compared with SOC, most trials included in our study were unblinded with low quality. Therefore, further well-designed studies are needed to investigate the safety issues of TCM.

Compared with previous relevant meta-analysis, a major strength of our study is the comprehensive search and analysis of effectiveness and safety profiles for all kinds of pharmacological treatments in a whole network with the largest number of studies and sample size. Furthermore, we included all pharmacological treatments recommended by several guidelines ${ }^{2-5}$, including TCM and CON_PLA as well as other treatments evaluated in previous meta-analysis. Meanwhile, placebo and SOC were separated as two treatment nodes in our evidence network, which could minimize bias due to potential placebo effect ${ }^{21}$. Additionally, we assessed the quality of evidence and incorporate it into explaining the results by the GRADE framework.

Several limitations, however, should be mentioned. First, most comparisons were assessed as low or very low quality in GRADE framework with wide confidence intervals owing to sparse data, which might restrict the interpretation of results. However, these data are still valuable and timely at this stage with no effective specific drugs for COVID-19. When more data of ongoing trials are available, we will update the analysis. Secondly, methodology of some included trials was poor. Nearly $80 \%$ of trials were not performed well in blinding or concealment allocation. Thus, this may introduce bias and results should be interpreted with caution. However, it might be difficult to conduct doubleblind trials for a contagious disease in some clinical situations. Finally, due to sparse data and unavailable access to original trial data, we could not perform detailed NMA subgroup analyses, meta-regression or individual patient data meta-analysis to properly address potentially relevant effect modifiers, such as age, disease severity and treatment duration.

\section{Conclusions}

Corticosteroids and TCM may reduce mortality and increase cure rate with no increased risk of OAEs compared with standard care. However, CQ_HD might increase the risk of mortality. CQ, IFN and other antiviral agents could increase the incidence of OAEs. A majority of trials are small-scale trials with important methodological limitations, and no definitive conclusion could be drawn for most treatments. The current evidence is generally uncertain with low quality and further high-quality trials are needed.

\section{Strengths And Limitations}

- Most comparisons were assessed as low or very low quality in GRADE framework with wide confidence intervals owing to sparse data. 
- Methodology of some included trials was poor, particularly in blinding or concealment allocation. Detailed NMA subgroup analyses, meta-regression or individual patient data meta-analysis could not be performed to properly address potentially relevant effect modifiers, such as age, disease severity and treatment duration.

- Comprehensive search and analysis of effectiveness and safety profiles for all kinds of pharmacological treatments in a whole network with the largest number of studies and sample size.

- We assessed the quality of evidence and incorporate it into explaining the results by the GRADE framework.

\section{Declarations}

\section{Acknowledgements}

We gratefully appreciate the excellent work for Xianming Cai, Shan Cai and Xiaoyu Tan (Peking University). Special thanks to their meticulous help with study selection.

\section{Funding}

This study is funded by National Natural Science Foundation of China (71673003), Special Project for Major Infectious Disease of Peking University Health Program of China (BMU2020HKYZX010) and National Key Technology R\&D Program of China (2020YFC0840800). The sponsor had no role in study design, data collection, data analysis, data interpretation, or writing of the report.

\section{Author Contributions}

FS and SYZ designed the study. FS, XYZ, JXZ and QXZ extracted the data, SSW, ZRY and QXZ evaluated the RCTs quality. SSW and FS assessed the quality of evidence by GRADE framework. XYZ and QXZ verified the data, FS and SSW analyzed the data. SSW drafted the manuscript. ZRY, FS and ZLZ revised the manuscript. FS and SYZ interpreted the results, incorporated comments for the co-authors and finalized the manuscript. All authors approved the final version of the paper.

\section{Conflicts of interests}

All authors have completed the ICMJE uniform disclosure form at www.icmje.org/coi_disclosure.pdf and declare no potential conflicts of interest relevant to this article.

\section{References}

1. World Health Organization (WHO). Available online: https://www.who.int/emergencies/diseases/novel-coronavirus-2019(accessed on 4 September 2020).

2. COVID-19 Treatment Guidelines Panel. Coronavirus Disease 2019 (COVID-19) Treatment Guidelines. National Institutes of Health. Available at https://www.covid19treatmentguidelines.nih.gov/ (accessed on 10 July 2020).

3. National Health Commission of the People's Republic of China. Chinese Clinical Guidance for COVID-19 Pneumonia Diagnosis and Treatment(8th edition). Available at

http://www.nhc.gov.cn/yzygj/s7653p/202008/0a7bdf12bd4b46e5bd28ca7f9a7f5e5a/files/a449a3e2e2c94d9a856d5faea2ffof94.pdf (accessed on 20 August 2020).

4. World Health Organization. Clinical management of COVID-19. Interim guidance, 27 May, 2020. https://www.who.int/publications/i/item/clinical-management-of-severe-acute-respiratory-infection-when-novel-coronavirus-(ncov)infection-is-suspected (accessed on 20 August 2020).

5. Rochwerg B, Agarwal A, Zeng L, Leo YS, Appiah JA, Agoritsas T, et al. Remdesivir for severe covid-19: a clinical practice guideline. BMJ. 2020 Jul 30;370:m2924. doi: 10.1136/bmj.m2924.

6. Sanders JM,Monogue ML, Jodlowski TZ, Cutrell JB. Pharmacologic Treatments for Coronavirus Disease 2019 (COVID-19): A Review. JAMA. 2020 Apr 13. doi: 10.1001/jama.2020.6019. Online ahead of print.

7. Yao TT, Qian JD, Zhu WY, Wang Y, Wang GQ. A systematic review of lopinavir therapy for SARS coronavirus and MERS coronavirus-A possible reference for coronavirus disease-19 treatment option. J Med Virol. 2020 Jun; 92(6):556-563. doi: 10.1002/jmv.25729. Epub 2020 Mar 12.

8. Das S, Bhowmick S, Tiwari S, Sen S. An Updated Systematic Review of the Therapeutic Role of Hydroxychloroquine in Coronavirus Disease-19 (COVID-19). Clin Drug Investig. 2020 Jul;40(7):591-601. doi: 10.1007/s40261-020-00927-1. 
9. Liu W, Zhou PX, Chen K, Ye ZK, Liu F, Li XT, et al. Efficacy and safety of antiviral treatment for COVID-19 from evidence in studies of SARS-CoV-2 and other acute viral infections: a systematic review and meta-analysis. CMAJ. 2020 Jul 6;192(27): E734-E744. doi: 10.1503/cmaj.200647. Epub 2020 Jun 3

10. Cortegiani A, Ingoglia G, Ippolito M, Giarratano A, Einav S. A systematic review on the efficacy and safety of chloroquine for the treatment of COVID-19. J Crit Care. 2020 Jun;57:279-283. doi: 10.1016/j.jcrc.2020.03.005. Epub 2020 Mar 10.

11. Halpin DMG, Singh D, Hadfield RM. Inhaled corticosteroids and COVID-19: a systematic review and clinical perspective. Eur Respir J. 2020 May 7;55(5):2001009. doi: 10.1183/13993003.01009-2020. Print 2020 May.

12. Rajendran K, Krishnasamy N, Rangarajan J, Rathinam J, Natarajan M, Ramachandran A. Convalescent plasma transfusion for the treatment of COVID-19: Systematic review. J Med Virol. 2020 May 1;10.1002/jmv.25961. doi: 10.1002/jmv.25961. Online ahead of print.

13. Ye ZK, Wang Y, Colunga-Lozano LE, Prasad M, Tangamornsuksan W, Rochwerg B, et al. Efficacy and safety of corticosteroids in COVID19 based on evidence for COVID-19, other coronavirus infections, influenza, community-acquired pneumonia and acute respiratory distress syndrome: a systematic review and meta-analysis. CMAJ. 2020 Jul 6;192(27):E756-E767. doi: 10.1503/cmaj.200645. Epub 2020 May 14.

14. Liu M, Gao Y, Yuan Y, Yang KL, Shi SZ, Zhang JH, et al. Efficacy and Safety of Integrated Traditional Chinese and Western Medicine for Corona Virus Disease 2019 (COVID-19): a systematic review and meta-analysis. Pharmacol Res. 2020 Aug;158:104896. doi: 10.1016/j.phrs.2020.104896. Epub 2020 May 11.

15. Xiong XJ, Wang PQ, Su KL, Cho WC, Xing YW. Chinese herbal medicine for coronavirus disease 2019: A systematic review and metaanalysis. Pharmacol Res. 2020 Jul 2;160:105056. doi: 10.1016/j.phrs.2020.105056. Online ahead of print.

16. Ang L, Song E, Lee HW, Lee MS. Herbal Medicine for the Treatment of Coronavirus Disease 2019 (COVID-19): A Systematic Review and Meta-Analysis of Randomized Controlled Trials. J Clin Med. 2020 May 23;9(5):1583. doi: 10.3390/jcm9051583.

17. Jin LR, Xu Y, Yuan H. Effects of four types of integrated Chinese and Western medicines for the treatment of COVID-19 in China: a NMA. Rev Assoc Med Bras (1992). 2020 Jun;66(6):771-777. doi: 10.1590/1806-9282.66.6.771. Epub 2020 Jul 20.

18. Siemieniuk RA, Bartoszko JJ, Ge L, Zeraatkar D, Izcovich A, Pardo-Hernandez H, et al. Drug treatments for covid-19: living systematic review and NMA. BMJ. 2020 Jul 30;370:m2980. doi: 10.1136/bmj.m2980.

19. Boutron I, Chaimani A, Devane D, et al. Interventions for preventing and treating COVID-19: protocol for a living mapping of research and a living systematic review. Zenodo [Preprint] 2020. https://zenodo.org/record/3744600\#.Xp8U0MgzZPY.

20. Juul S, Nielsen N, Bentzer P, et al. Interventions for treatment of COVID-19: a protocol for a living systematic review with NMA including individual patient data (The LIVING Project). Syst Rev 2020;9:108. doi:10.1186/s13643-020-01371-0.

21. Guevarra DA, Moser JS, Wager TD, Kross E. Placebos without deception reduce self-report and neural measures of emotional distress. Nat Commun. 2020 Jul 29;11(1):3785. doi: 10.1038/s41467-020-17654-y.

22. Higgins JPT, Thomas J, Chandler J, Cumpston M, Li T, Page MJ, Welch VA (editors). Cochrane Handbook for Systematic Reviews of Interventions version 6.0 (updated July 2019). Cochrane, 2019. Available from training.cochrane.org/handbook (accessed 10 August, 2020)

23. Salanti G, Del Giovane C, Chaimani A, Caldwell DM, Higgins JP. Evaluating the quality of evidence from a NMA. PLoS One. 2014; 9 (7): e99682. doi: 10.1371/journal.pone.0099682. eCollection 2014.

24. Sweeting MJ, Sutton AJ, Lambert PC. What to add to nothing? Use and avoidance of continuity corrections in meta-analysis of sparse data. Statistics in Medicine 2004; 23: 1351-75.

25. Salanti G. Indirect and mixed-treatment comparison, network, or multipletreatments meta-analysis: Many names, many benefits, many concerns for the next generation evidence synthesis tool. Res Synth Methods. 2012; 3(2): 80-97. doi: 10.1002/jrsm.1037.

26. Veroniki AA, Straus SE, Fyraridis A, Tricco AC. The rank-heat plot is a novel way to present the results from a NMA including multiple outcomes. J Clin Epidemiol. 2016 Aug;76:193-9. doi: 10.1016/j.jclinepi.2016.02.016.

27. Higgins JP, Jackson D, Barrett JK, Lu G, Ades AE, White IR. Consistency and inconsistency in networkmeta-analysis: concepts and models for multi-arm studies. Res Synth Methods. 2012; 3(2): 98-110. doi: 10.1002/jrsm.1044.

28. Chaimani ASG. Visualizing assumptions and results in NMA: The network graphs package. Stata J. 2015; 15: 905-50.

29. Z Zhao, F Zhang, M Xu, K Huang, W Zhong, W Cai, et al. Description and clinical treatment of an early outbreak of severe acute respiratory syndrome (SARS) in Guangzhou, PR China. J Med Microbiol. 2003 Aug;52(Pt 8):715-720. doi: 10.1099/jmm.0.05320-0.

30. Guvenmez O, Keskin H, Ay B, Birinci S, Kanca MF. The comparison of the effectiveness of lincocin ${ }^{\circledR}$ and azitro ${ }^{\circledR}$ in the treatment of covid-19-associated pneumonia: A prospective study. J Popul Ther Clin Pharmacol. 2020 Jun 3;27(S Pt 1):e5-e10. doi: 
10.15586/jptcp.v27iSP1.684.

31. Molina JM, Delaugerre C, Le Goff J, Mela-Lima B, Ponscarme D, Goldwirt L, et al. No evidence of rapid antiviral clearance or clinical benefit with the combination of hydroxychloroquine and azithromycin in patients with severe COVID-19 infection. Med Mal Infect. 2020. Epub 2020/04/03.

32. Cao B, Wang Y, Wen D, Liu W, Wang J, Fan G, et al. A Trial of Lopinavir-Ritonavir in Adults Hospitalized with Severe Covid-19. N Engl J Med. 2020. Epub 2020/03/19.

33. Wang YM, Zhang DY, Du GH, Du RH, Zhao JP, Jin Y, et al. Remdesivir in adults with severe COVID-19: a randomised, double-blind, placebo-controlled, multicentre trial. Lancet. 2020 May 16;395(10236):1569-1578. doi: 10.1016/S0140-6736(20)31022-9.

34. Tang W, Cao Z, Han M, Wang Z, Chen J, Sun W, et al. Hydroxychloroquine in patients with mainly mild to moderate coronavirus disease 2019: open label, randomised controlled trial. BMJ. 2020;369:m1849.

35. Geleris J, Sun Y, Platt J, Zucker J, Baldwin M, Hripcsak G, et al. Observational Study of Hydroxychloroquine in Hospitalized Patients with Covid-19. N Engl J Med. 2020. Epub 2020/05/08.

36. Horby P, Lim WS, Emberson J, et al. Effect of Dexamethasone in Hospitalized Patients with COVID-19: Preliminary Report. medRxiv [Preprint] 2020. doi:10.1101/2020.06.22.20137273

37. Ni YN, Chen G, Sun J, Liang BM, Liang ZA. The effect of corticosteroids on mortality of patients with influenza pneumonia: a systematic review and meta-analysis. Crit Care. 2019;23(1):99. doi:10.1186/s13054-019-2395-8

38. Wu C, Chen X, Cai Y, Xia J, Zhou X, Xu S, et al. Risk factors associated with acute respiratory distress syndrome and death in patients with coronavirus disease 2019 pneumonia inWuhan, China. JAMA Intern Med. Published online March 13, 2020.

39. Luo H, Tang QL, Shang YX, Liang SB, Yang M, Robinson N, et al. Can Chinese Medicine Be Used for Prevention of Corona Virus Disease 2019 (COVID-19)? A Review of Historical Classics, Research Evidence and Current Prevention Programs. Chin J Integr Med. 2020 Apr;26(4):243-250. doi: 10.1007/s11655-020-3192-6.

40. Shi Y, Wang XP, Bai JQ, Long KH. Study on the pharmacological action of antibacterial and antiviral by Forsythia Fruit (Lianqiao, Fructus Forsythiae Suspensae), Mod. Chin. Med. 15 (2013) 950-953.

41. Huang CF, Lin SS, Liao PH, Young SC, Yang CC. The immunopharmaceutical effects and mechanisms of herb medicine, Cell. Mol. Immunol. 5 (2008) 23-31.

42. Du C, Zheng K, Bi C, Dong T, Lin H, Tsim K, et al. An ancient Chinese herbal decoction, induces gene expression of anti-viral proteins and inhibits neuraminidase activity. Phytother Res 2015;29:656-661.

43. Liu L, Lei N, Lin Q, Wang L, Yan H, Duan X. The effects and mechanism of Yinqiao Powder on upper respiratory tract infection. Int $J$ Biotech Wellness Indust 2015;4:57-60.

\section{Figures}




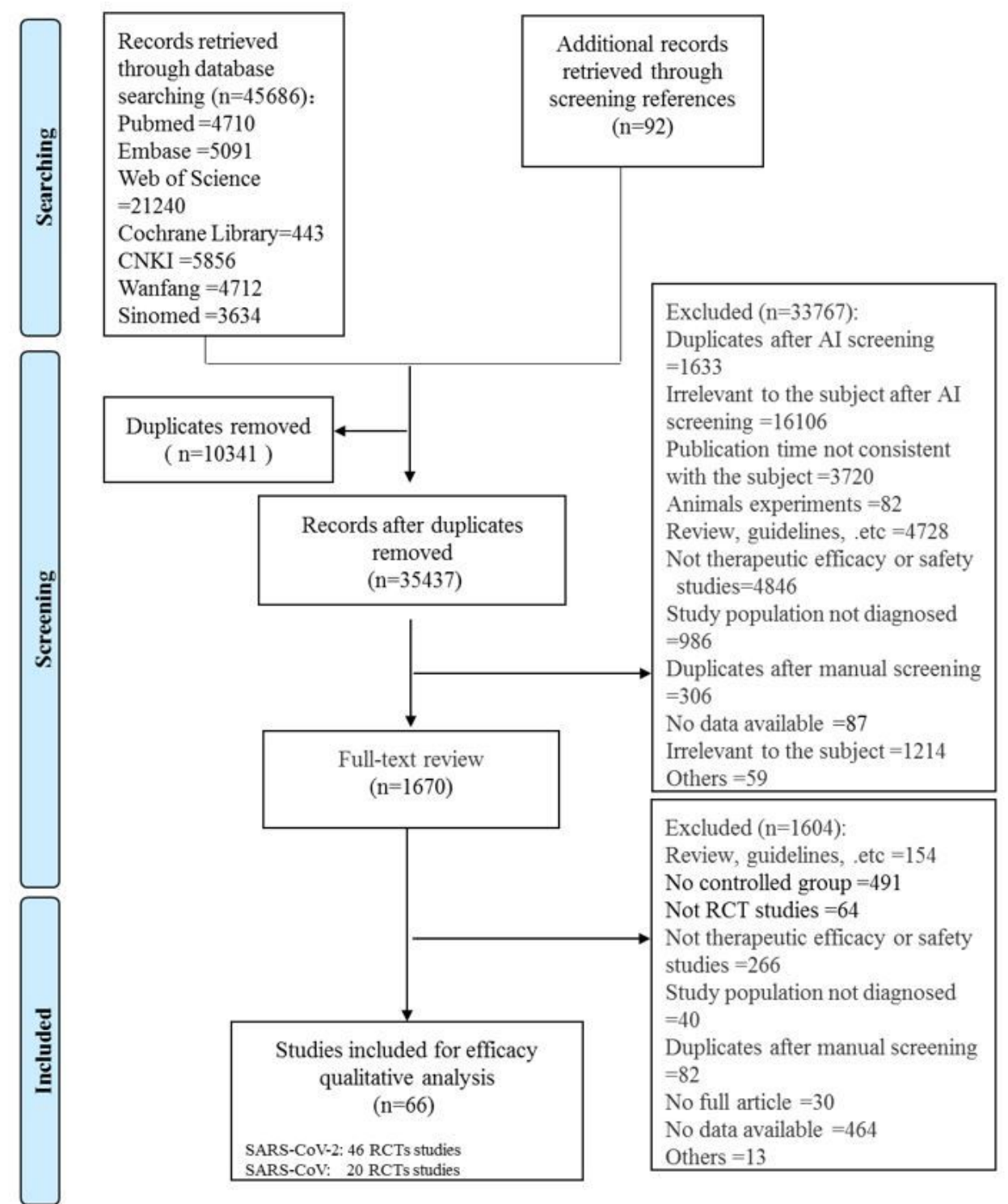

Figure 1

Flow chart of studies considered for inclusion. 


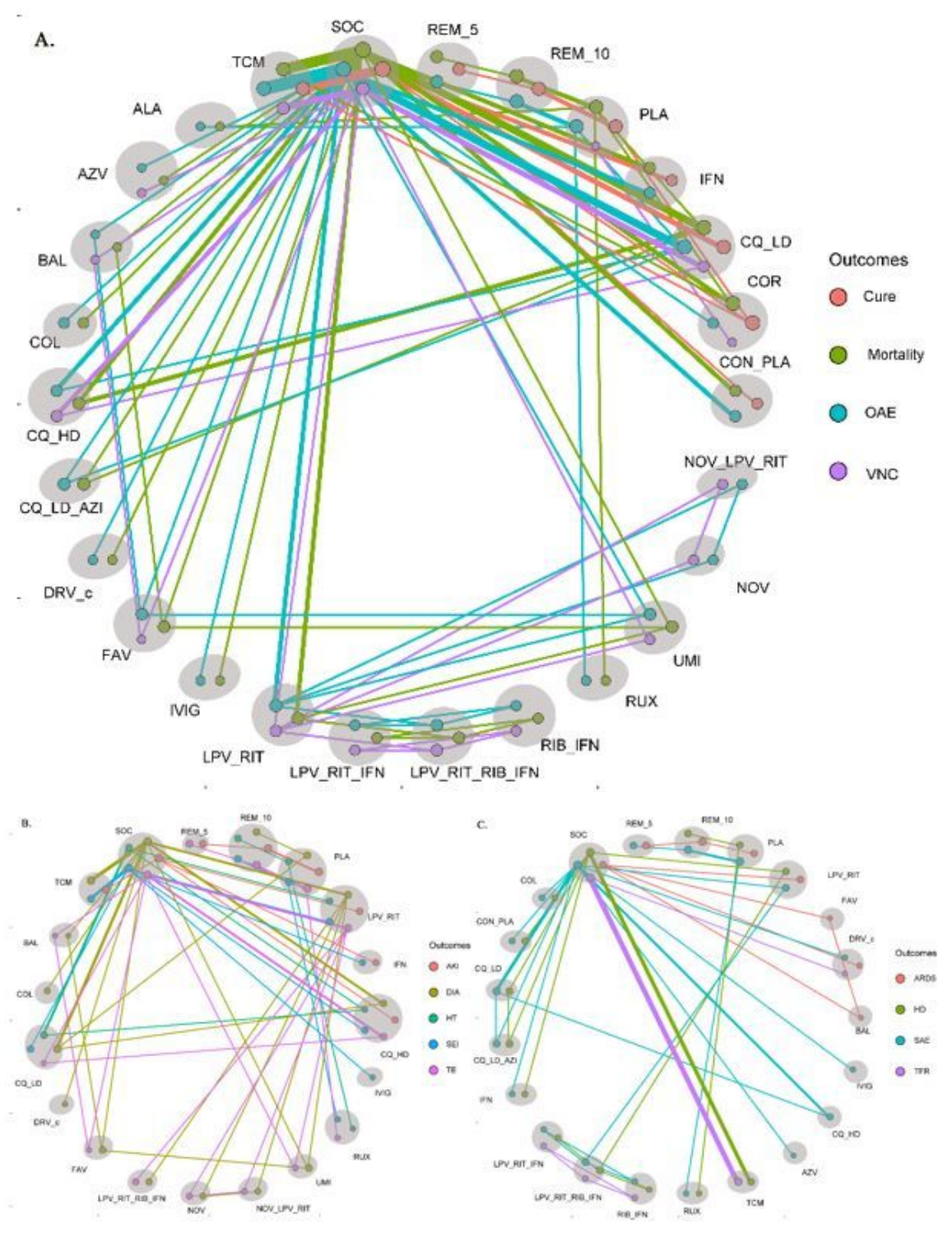

Figure 2

Evidence structure of eligible comparisons for network meta-analysis. A. Primary outcomes including mortality, cure, VNC and OAE; B. Secondary outcomes including DIA, AKI, TE, SEl and HF; C. Secondary outcomes including ARDS, SAE, HD and TFR. Lines connect the interventions that have been studied in head-to-head (direct) comparisons in the eligible RCTs. The width of the lines represents the cumulative number of RCTs for each pairwise comparison and the size of every node is proportional to the number of randomized participants (sample size). VNC: viral negative conversion; OAE: overall adverse events; DIA: diarrhea; AKI: acute kidney injury; TE: Transaminase elevation; SEl: secondary infection; HF: heart failure; ARDS: acute respiratory dyspnea syndrome; SAE: serious adverse events; HD: Hospitalization duration; TFR: Time to fever resolution; SOC: Standard of care; ALA: a-Lipoic acid; BAL: Baloxavir marboxil; CON_PLA: Convalescent plasma; CQ_HD: High Doses of Chloroquine; CQ_LD: Low Doses of Chloroquine; FAV: Favipiravir; COR: Cortcosteroids; IFN: Interferon- $\beta-1$ a; LPV_RIT: Lopinavir/Ritonavir; LPV_RIT_IFN: Lopinavir/ritonavir+Interferon- $\beta-1$ b; LPV_RIT_RIB_IFN: Lopinavir/ritonavir+Ribavirin+Interferon- $\beta-1$ b; RIB_IFN: Ribavirin+Interferon- $\beta$-1b; PLA: Placebo; REM_10: Remdesivir 10 mg/day; REM_5: Remdesivir 5 mg/day; RUX: Ruxolitinib; TCM: Traditional Chinese Medicine; UMI: Umifenovir; NOV: Novaferon; NOV_LPV_RIT: Novaferon + Lopinavir/Ritonavir; CQ_LD_AZI: Low Doses of Chloroquine+Azithromycin; AZV: Azvudine; RIB_CEFs: Ribavirin+Cefperazone-Sulbacta; IVIG: Octagam 10\%; DRV_c; Single-tablet regimen containing $800 \mathrm{mg}$ of darunavir and $150 \mathrm{mg}$ of cobicistat; LEV_AZI_IFN_COR: Levofloxacin+Azithromycin+IFN-a+Methylprednisolone; COL: colchicine; QUI_AZI_IFN_COR: Quinolone+Azithromycin+IFNa+Methylprednisolone. 
A.

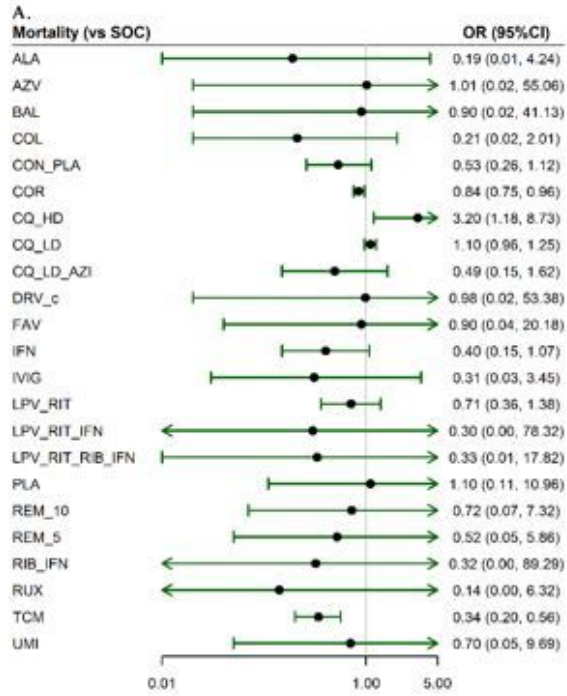

C.

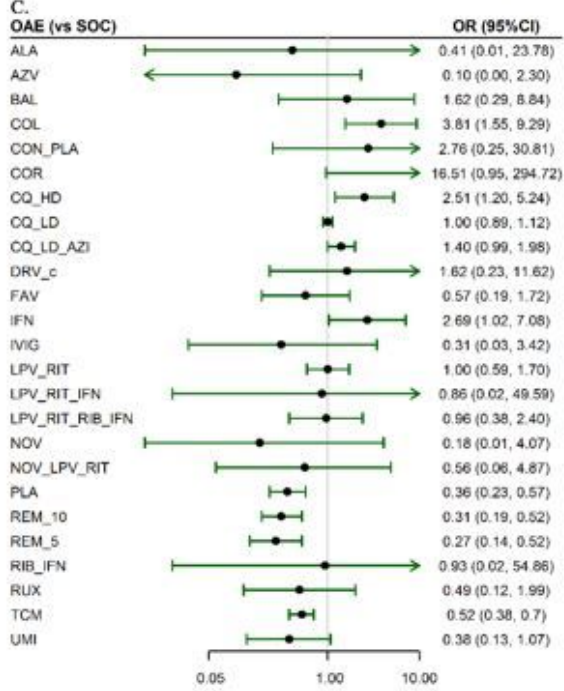

B.

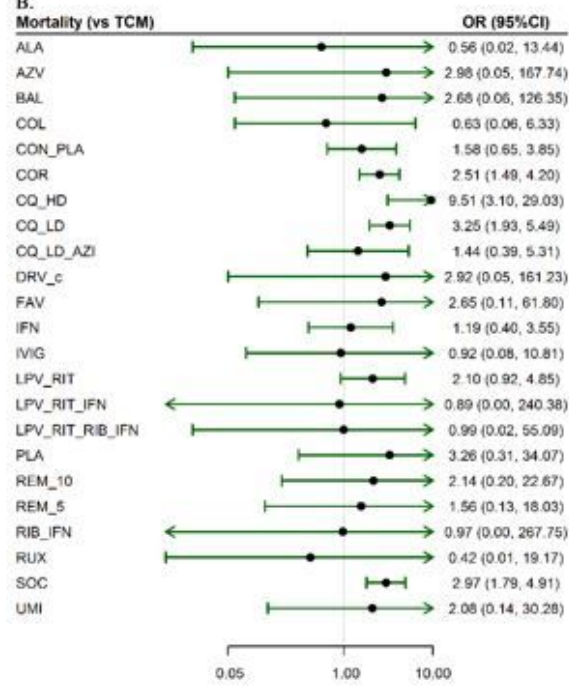

D.

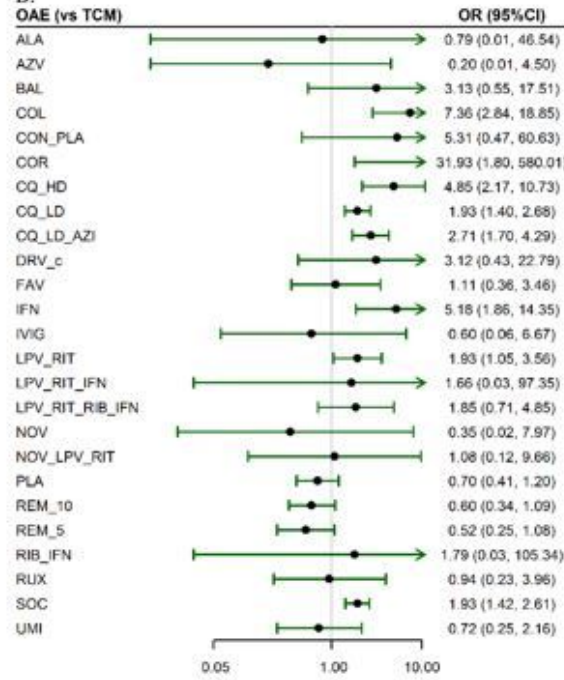

Figure 3

Results of network meta-analysis for mortality and OAE. A. Mortality compared with SOC; B. Mortality compared with TCM; C. OAE compared with SOC; D. OAE compared with TCM. OAE: overall adverse events; OR: odds ratio; SOC: Standard of care; ALA: a-Lipoic acid; BAL: Baloxavir marboxil; CON_PLA: Convalescent plasma; CQ_HD: High Doses of Chloroquine; CQ_LD: Low Doses of Chloroquine; FAV: Favipiravir; COR: Cortcosteroids; IFN: Interferon- $\beta$-1a; LPV_RIT: Lopinavir/Ritonavir; LPV_RIT_IFN: Lopinavir/ritonavir+Interferon- $\beta$-1b;

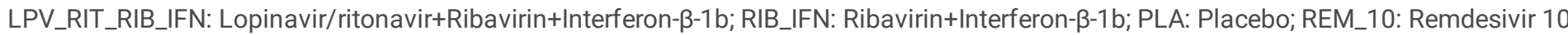
mg/day; REM_5: Remdesivir 5 mg/day; RUX: Ruxolitinib; TCM: Traditional Chinese Medicine; UMI: Umifenovir; NOV: Novaferon; NOV_LPV_RIT: Novaferon + Lopinavir/Ritonavir; CQ_LD_AZI: Low Doses of Chloroquine+Azithromycin; AZV: Azvudine; IVIG: Octagam 10\%; DRV_c; Single-tablet regimen containing $800 \mathrm{mg}$ of darunavir and $150 \mathrm{mg}$ of cobicistat; COL: colchicine. 
A.

Cure

Compared with SOC

CON_PLA

COR

CQ_LD

IFN

PLA

REM_10

REM_5

TCM

Compared with TCM

CON_PLA

COR

CQ_LD

IFN

PLA

REM_10

REM_5

SOC
$\mathrm{OR}(95 \% \mathrm{Cl})$

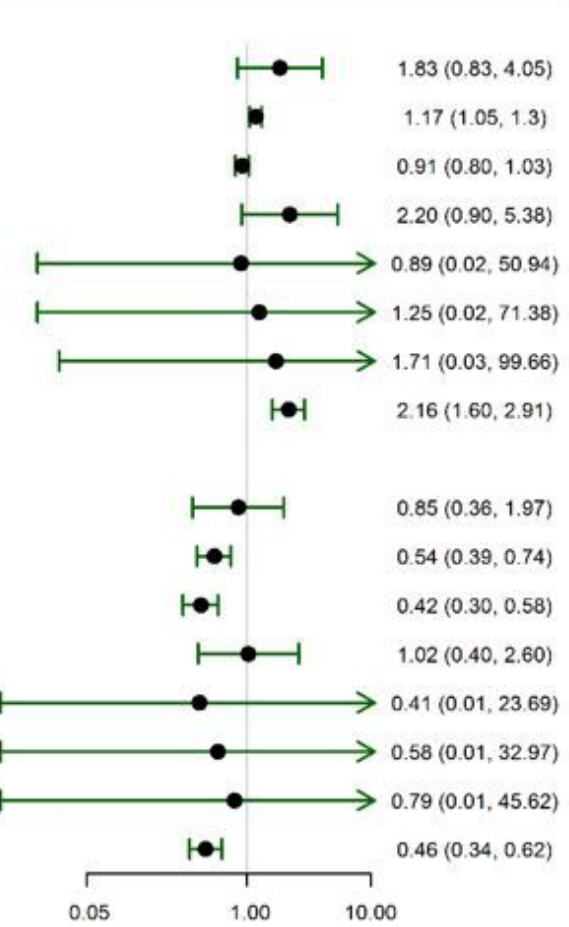

B.

VNC $\quad$ OR(95\%Cl)

Compared with SOC

AZV

BAL

CQ_HD

CQ_LD

FAV

LPV_RIT

LPV_RIT_IFN

LPV_RIT_RIB_IFN

NOV

NOV_LPV_RIT

RIB_IFN

TCM

UMI

Compared with TCM

AZV

BAL

CQ_HD

CQ_LD

FAV

LPV_RIT

LPV_RIT_IFN

LPV_RIT_RIB_IFN

NOV

NOV_LPV_RIT

RIB_IFN

$\mathrm{SOC}$

UMI
$.03(0.00,0.69)$

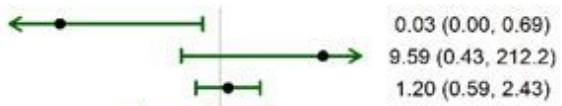

$0.77(0.18,3.21)$

$6.82(0.28,162.79)$

$0.56(0.13,2.41)$

$0.43(0.04,5.09)$

$0.76(0.11,5.10)$

$0.46(0.08,2.72)$

$0.26(0.04,1.58)$

$1.63(0.14,18.42)$

$0.68(0.44,1.05)$

$0.30(0.06,1.54)$

$0.05(0.00,1.07)$

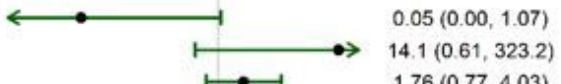

$1.76(0.77,4.03)$

$1.13(0.26,5.04)$

$10.03(0.41,248.52)$

$0.82(0.18,3.78)$

$0.64(0.05,7.75)$

$1.12(0.16,7.9)$

$0.68(0.11,4.27)$

$0.38(0.06,2.45)$

$2.40(0.21,28.24)$

$1.47(0.95,2.28)$

$0.45(0.08,2.41)$

Figure 4

Results of network meta-analysis for cure and VNC compared with SOC and TCM. A. Cure; B. VNC. VNC: viral negative conversion; OR: odds ratio; SOC: Standard of care; ALA: a-Lipoic acid; BAL: Baloxavir marboxil; CON_PLA: Convalescent plasma; CQ_HD: High Doses of Chloroquine; CQ_LD: Low Doses of Chloroquine; FAV: Favipiravir; COR: Cortcosteroids; IFN: Interferon- $\beta-1 a$; LPV_RIT: Lopinavir/Ritonavir; LPV_RIT_IFN: Lopinavir/ritonavir+Interferon- $\beta$-1b; LPV_RIT_RIB_IFN: Lopinavir/ritonavir+Ribavirin+Interferon- $\beta$-1b; RIB_IFN:

Ribavirin+Interferon- $\beta$-1b; PLA: Placebo; REM_10: Remdesivir 10 mg/day; REM_5: Remdesivir 5 mg/day; RUX: Ruxolitinib; TCM: Traditional Chinese Medicine; UMI: Umifenovir; NOV: Novaferon; NOV_LPV_RIT: Novaferon + Lopinavir/Ritonavir; CQ_LD_AZI: Low Doses of Chloroquine+Azithromycin; AZV: Azvudine; IVIG: Octagam 10\%; DRV_c; Single-tablet regimen containing 800 mg of darunavir and 150 mg of cobicistat; COL: colchicine. 
A.
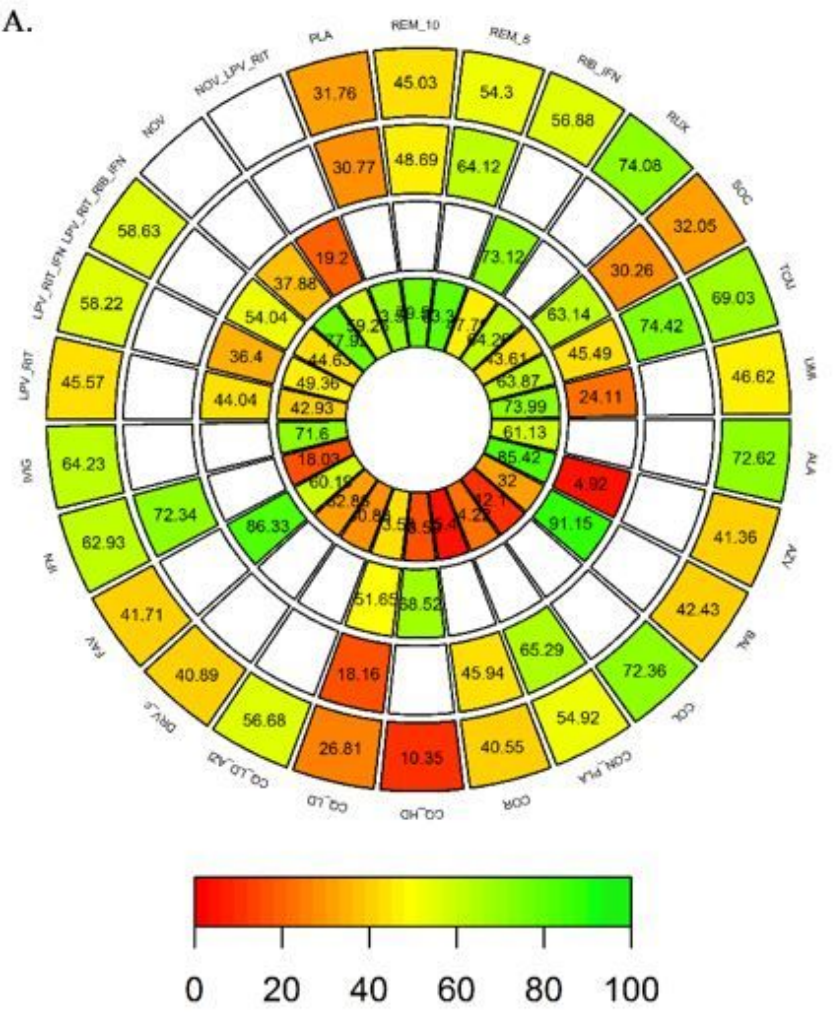

B.
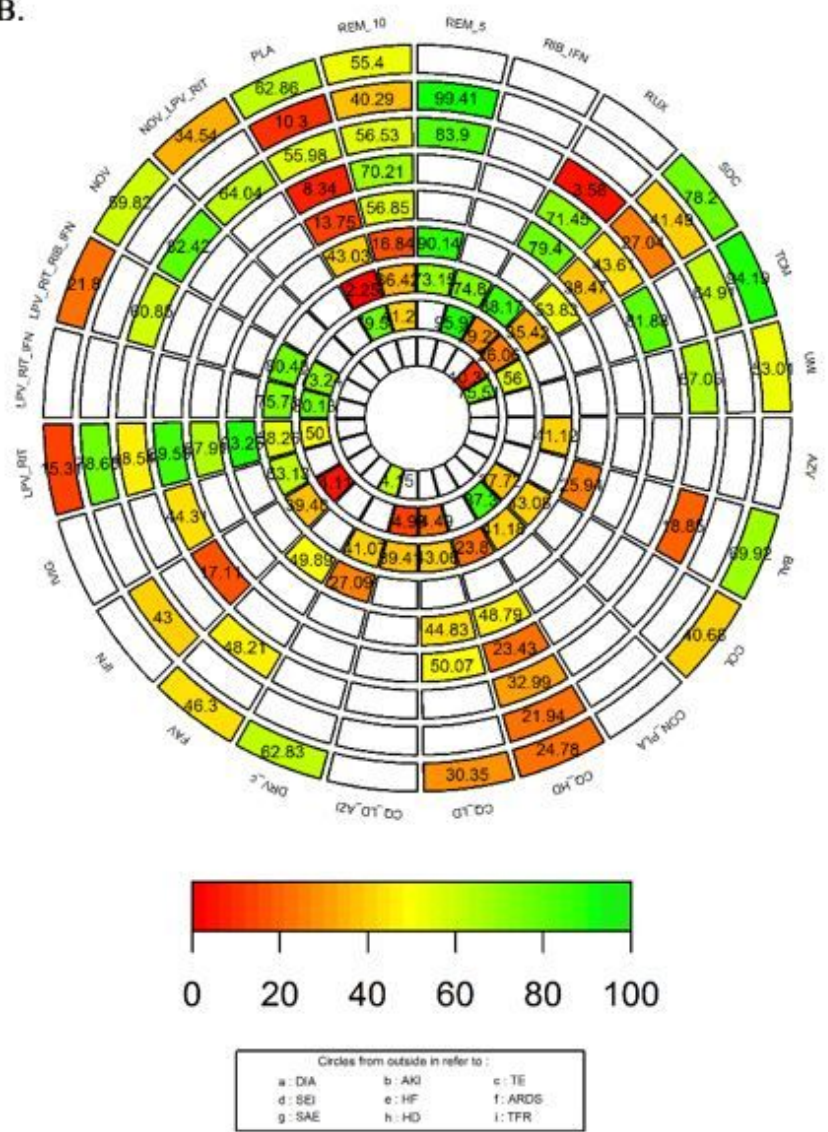

\section{Figure 5}

Rank heat plots for each outcome. A. Primary outcomes (Mortality, Cure, VNC, OAE); B. Secondary outcomes (DIA, AKI, TE, SEI, HF, ARDS, SAE, HD and TFR). Rank-heat plot of 19 and 17 treatments (presented in radii) for 4 primary outcomes and 9 secondary outcomes (presented in concentric circles). Each sector is colored according to SUCRA value of corresponding treatment and outcome using the transformation of three colors: red (0\%), yellow (50\%), and green (100\%). VNC: viral negative conversion; OAE: overall adverse events; DIA: diarrhea; AKI: acute kidney injury; TE: Transaminase elevation; SEl: secondary infection; HF: heart failure; ARDS: acute respiratory dyspnea syndrome; SAE: serious adverse events; HD: Hospitalization duration; TFR: Time to fever resolution; SOC: Standard of care; ALA: a-Lipoic acid; BAL: Baloxavir marboxil; CON_PLA: Convalescent plasma; CQ_HD: High Doses of Chloroquine; CQ_LD: Low Doses of Chloroquine; FAV: Favipiravir; COR: Cortcosteroids; IFN: Interferon- $\beta-1$ a; LPV_RIT: Lopinavir/Ritonavir; LPV_RIT_IFN: Lopinavir/ritonavir+Interferon- $\beta$-1b; LPV_RIT_RIB_IFN: Lopinavir/ritonavir+Ribavirin+Interferon-B-1b; RIB_IFN: Ribavirin+Interferon- $\beta-1 b$; PLA: Placebo; REM_10: Remdesivir 10 mg/day; REM_5: Remdesivir 5 mg/day; RUX: Ruxolitinib; TCM: Traditional Chinese Medicine; UMI: Umifenovir; NOV: Novaferon; NOV_LPV_RIT: Novaferon + Lopinavir/Ritonavir; CQ_LD_AZI: Low Doses of Chloroquine+Azithromycin; AZV: Azvudine; RIB_CEFs: Ribavirin+Cefperazone-Sulbacta; IVIG: Octagam 10\%; DRV_c; Single-tablet regimen containing $800 \mathrm{mg}$ of darunavir and $150 \mathrm{mg}$ of cobicistat; LEV_AZI_IFN_COR: Levofloxacin+Azithromycin+IFN-a+Methylprednisolone; COL: colchicine; QUI_AZI_IFN_COR: Quinolone+Azithromycin+IFN-a+Methylprednisolone.

\section{Supplementary Files}

This is a list of supplementary files associated with this preprint. Click to download.

- 4Appendix.docx

- PRISMA2009ChecklistMSWord.doc 